\title{
The Care Processes for Acute Cholecystitis According to Clinical Practice Guidelines Based on the Japanese Administrative Database
}

\author{
Atsuhiko Murata, ${ }^{1}$ Kohji Okamoto, ${ }^{2}$ Shinya Matsuda, ${ }^{1}$ Kazuaki Kuwabara, ${ }^{3}$ \\ Yukako Ichimiya, ${ }^{1}$ Yasufumi Matsuda, ${ }^{1}$ Tatsuhiko Kubo, ${ }^{1}$ Yoshihisa Fujino, ${ }^{1}$ \\ Kenji Fujimori ${ }^{4}$ and Hiromasa Horiguchi ${ }^{5}$
}

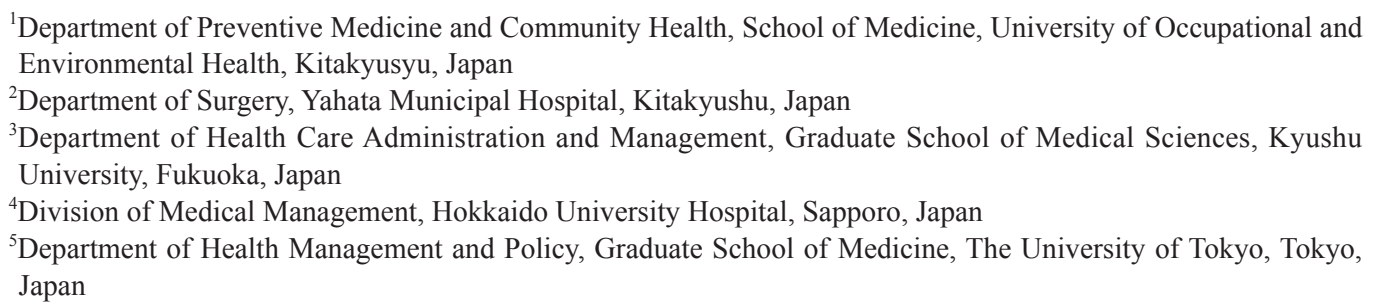

Acute cholecystitis is one of the most frequently encountered conditions in daily practice in Japan. However, there is a shortage of detailed data about treatments that have been performed according to the clinical practice guidelines (CPGs) for acute cholecystitis. We therefore examined the management of acute cholecystitis for adherence to the appropriate CPGs using the Japanese administrative database associated with the Diagnosis Procedure Combination (DPC) system. We collected data from 6,070 patients with acute cholecystitis, examining for the application of four recommended treatments (administration of antimicrobial drugs and nonsteroidal anti-inflammatory drugs (NSAIDs) and performance of early and laparoscopic cholecystectomy). The patients were classified according to the procedures documented for each case: no gallbladder drainage $(n=4,333)$, gallbladder drainage without supportive care (ventilation or hemodiafiltration or the use of vasopressor) $(n=1,591)$ and gallbladder drainage and supportive care $(n=146)$. Multiple logistic regression models revealed that patients with gallbladder drainage without supportive care and those with gallbladder drainage and supportive care significantly higher received administration of antimicrobial drugs and NSAIDs, while these patients underwent less early or laparoscopic cholecystectomy than did patients without gallbladder drainage, after adjusting for potential confounding effects of the clinical variables. This study demonstrated that there were various differences with regard to the performance of recommended treatments between the levels of procedures required for acute cholecystitis. In addition, this administrative database was a feasible tool for the evaluation of care processes and will provide useful information contributing to improved quality of medical care.

Keywords: acute cholecystitis; administrative database; care processes; clinical practice guidelines; diagnosis procedure combination

Tohoku J. Exp. Med., 2012, 227 (4), 237-244. C 2012 Tohoku University Medical Press

Acute cholecystitis is an acute inflammatory disease of the gallbladder caused mainly by bacterial infection (Barie and Eachempati 2010). According to a comprehensive survey of living conditions of the Japanese people on health and welfare, the number of patients with acute cholecystitis has increased by approximately three times between the 1970s and 1990s in Japan (Kimura et al. 2007). Therefore, this medical condition is still one of the most frequently encountered conditions in daily practice in Japan.

Regarding the treatments for acute cholecystitis, antimicrobial therapy remains the mainstay of therapy for acute cholecystitis (Westphal and Brogard 1999). However, gallbladder drainage, either as endoscopic nasogallbladder drainage (ENGBD) or percutaneous transhepatic gallbladder drainage (PTGBD), has been shown to be useful when the patient has not improved or if there is worsening with

Received April 17, 2012; revision accepted for publication June 22, 2012. doi: 10.1620/tjem.227.237

Correspondence: Atsuhiko Murata, M.D., Department of Preventive Medicine and Community Health, School of Medicine, University of Occupational and Environmental Health, 1-1 Iseigaoka, Yahatanishi-ku, Kitakyushu 807-8555, Japan.

e-mail: amurata@med.uoeh-u.ac.jp 
antimicrobial therapy (Akhan et al. 2002; Toyota et al. 2006). In addition, cholecystectomy, especially the laparoscopic procedure, has been widely accepted as an effective treatment for acute cholecystitis (Zacks et al. 2002).

To establish standardization of these treatment processes for acute cholecystitis, clinical practice guidelines (CPGs), named the Tokyo Guidelines, were prepared on the basis of the best available evidence on all aspects of the diagnosis and treatment of acute cholecystitis in 2007 (Takada et al. 2007). These CPGs are the world's first international guidelines for the clinical management of acute cholecystitis and have substantially contributed to the care of patients with acute cholecystitis. Some recent studies have reported that the CPGs can help clinicians make the correct management decisions for patients with acute cholecystitis (Lee et al. 2010; Yokoe et al. 2011), suggesting that the CPGs have been useful and can be widely accepted among many doctors.

However, there have been no studies examining the degree to which appropriate treatments recommended by the CPGs have occurred in actual medical practice, based on large-scale patient data. In addition, there have been no studies where an administrative database has been utilized to evaluate the care processes given compared to those recommended by the CPGs for acute cholecystitis in community-based facilities. Such an evaluation could provide useful information for the quality of medical care in the future, as well as an assessment of present medical treatment for acute cholecystitis. In this study, we evaluated the care processes given patients with acute cholecystitis according to the CPGs. This was achieved by using the national administrative database developed in a Japanese case-mix system project, called the Diagnosis Procedure Combination (DPC).

\section{Methods}

Administrative database associated with the DPC system

The health care system in Japan has been facing serious financial problems because of a rapidly ageing society, costly innovations in medical technology, and longer patient hospitalizations. To solve these problems, the Ministry of Health, Labour and Welfare and its affiliated research institute have started research on the feasibility of using the Japanese case-mix classification system as a tool for standardized medical profiling and payment (Matsuda 2008). As a result, Japanese case-mix projects based on the DPC system were introduced to 82 academic hospitals ( 80 university hospitals, the National Cancer Centre, and the National Cardiovascular Centre) in 2003 (Murata et al. 2010, 2011, 2012). Insurance reimbursement using the DPC system is now very prevalent in Japan, and the administrative database of the DPC system has increased the representation of acute care hospitals. In 2008, 2,120,170 hospitalization records were gathered in the administrative database of the DPC system. These data were collected from hospitals participating in the survey, and 855 out of the 1,557 hospitals agreed to participate in the survey in 2008, which represented approximately $40 \%$ of acute inpatients during that time (Kubo et al. 2011).

The administrative database associated with the DPC system includes each patient's discharge summary and claim information, including principal diagnosis, and comorbidities and complications during hospitalization (Murata et al. 2010, 2011, 2012; Ichimiya et al. 2011). These data are coded in accordance with the International Classification of Diseases and Injuries (ICD)-10th edition. The database also contains detailed medical information, including all surgical or interventional procedures and medications that have been indexed in the original Japanese codes. These codes are determined by the Ministry of Health, Labour and Welfare of Japan. The database also includes the quantity and date of all care delivered on a daily basis (Murata et al. 2010, 2011, 2012).

\section{Study setting}

We selected 6,080 patients with acute cholecystitis from 777 DPC participating hospitals (68 academic and 709 community hospitals) between April and December of 2008. These hospitals are spread throughout Japan and play a leading role in providing acute care medicine, advancing medical research, and educating students and medical residents (Murata et al. 2010, 2011, 2012).In the present study, acute cholecystitis was defined as code K810 in the ICD-10th. For this analysis, we excluded 10 patients less than 15 years of age because the causes of pediatric acute cholecystitis are quite different from those of adult acute cholecystitis (e.g., serious burns, metabolic diseases or bone marrow transplantation) (Yasuda et al. 2007).

The use of DPC data were permitted by all institutions and hospitals that provided detailed data. The research protocol of the study was approved by the ethics committee of medical care and research of the University of Occupational and Environmental Health, Kitakyushu, Japan.

Selected recommended treatments and classification of acute cholecystitis

The recommended treatments selected in the CPGs for acute cholecystitis are shown in Table 1. There were four recommended treatments (recommendation A; Yamashita et al. 2007; Yoshida et al. 2007). These recommended treatments have been regarded as the procedures that should be performed for patients with acute cholecystitis.

We classified the patient groups by severity of acute cholecystitis, defined in the CPGs as grades I (mild), II (moderate) and III

Table 1. Selected recommended treatments for acute cholecystitis in the clinical practice guidelines.

\section{Recommendation A}

(1) Antimicrobial agents should be administered to patients diagnosed with acute cholecystitis.

(2) Administration of nonsteroidal anti-inflammatory drugs (NSAIDs) to patients with an attack of biliary colic is recommended, to prevent the onset of acute cholecystitis.

(3) Cholecystectomy is preferable early after admission.

(4) Laparoscopic cholecystectomy is preferable to open cholecystectomy. 
(severe) (Mayumi et al. 2007). The CPG flowcharts recommend treatment management according to severity (i.e., mild acute cholecystitis does not require gallbladder drainage; moderate cases require gallbladder drainage without organ support; severe cases require both gallbladder drainage and organ support) (Mayumi et al. 2007). Therefore, we defined each patient's acute cholecystitis group by the procedures performed for each patient, referring to these flowcharts: patients without gallbladder drainage $(n=4,333)$, patients with gallbladder drainage, but not supportive care such as ventilation or hemodiafiltration or the use of vasopressors [dopamine, dobutamine, noradrenalin or adrenalin] $(n=1,591)$ and patients with both gallbladder drainage and supportive care $(n=146)$. These classifications were confirmed for every patient using the DPC database, not by selfreport.

\section{Study variables}

Study variables used were age, sex, chronic co-morbid conditions, hospital type, use of ambulance transportation and intensive care unit (ICU), length of stay (LOS), in-hospital mortality, frequency of intravenous antimicrobial therapy and administration of nonsteroidal anti-inflammatory drugs (NSAIDs), kinds of antimicrobial drugs and NSAIDs, number of days from admission to cholecystectomy, kinds of cholecystectomy (open or laparoscopic procedures) and frequency of bile culture in this study.

The CPGs have defined elderly patients as those of age 75 years and more (Yasuda et al. 2007). Therefore, patients were stratified by age into two groups: $<75$ years of age and $\geq 75$ years of age. To assess the severity of chronic comorbid conditions, we employed the Charlson Comorbidity Index (CCI), which has been widely used for recording comorbidity and validated in various studies (Quan et al. 2005). CCI was expressed as the score of comorbid conditions and was initially evaluated as a continuous variable. However, categorical variables defining three categories of severity of comorbidity were created to simplify the presentation of the results: 0 , mild; 1 , moderate; and 2 or more, severe. Hospital type was classified as academic or community. For the selection of antimicrobial drugs for acute cholecystitis, we referred to the lists of antimicrobial drugs recommended in the CPGs. The use of first-generation cephalosporins or widespectrum penicillin/lactamase inhibitors is recommended in mild acute cholecystitis whereas second-generation cephalosporins or wide-spectrum penicillin/lactamase inhibitors are recommended in moderate acute cholecystitis. For patients with severe acute cholecystitis, third- and fourth-generation cephalosporins, monobactams, fluoroquinolones or carbapenems are recommended (Yoshida et al.
2007). Because there is no definition of early cholecystectomy in the CPGs, this was defined as cholecystectomy that had been performed within four days after admission, as described in previous studies (Navez, et al. 2001; Kitano et al. 2002).

\section{Main outcome measures for appropriate treatments according to the $C P G s$}

The recommended treatments for acute cholecystitis that correspond to each patient group are shown in Table 2. In this study, we further divided patient groups according to the presence of cholecystectomy and examined for the application of recommended treatments followed for each patient (for example, patients without gallbladder drainage and cholecystectomy were evaluated using 2 recommendations [items (1) (2) in Table 1] while those with gallbladder drainage, supportive care and cholecystectomy were evaluated using all 4 recommendations [items (1) - (4) in Table 1]).

\section{Statistical analysis}

The categorical data were compared using the chi-square test for categorical variables and the one-way factorial analysis of variance (ANOVA) and the Kruskal-Wallis tests for continuous variables. In additional analyses, multiple logistic regression models were used to identify the impact of the individual effect of patient acute cholecystitis group on each recommended treatment. We addressed potential confounding due to variation in the case mix by controlling for the severity of chronic co-morbid conditions and additional variables related to the adherence to the CPGs, such as age, sex, hospital type, use of ambulance transportation and ICU.

All statistical analyses were performed using the STATA statistical software package version 11.0 (Stata Corporation, College Station, TX, USA). A value of $p<0.05$ was considered significant.

\section{Results}

We examined a total of 6,070 patients in 777 hospitals, comprising 4,333 patients without gallbladder drainage, 1,591 patients with gallbladder drainage and without supportive care and 146 those with both gallbladder drainage and supportive care. The mean LOS in all patients was $20.2 \pm 18.2$ days $(19.7 \pm 18.7$ days for patients without cholecystectomy and $21.1 \pm 17.3$ days for patients with cholecystectomy) and the in-hospital mortality after admission was $2.1 \%$ (2.9\% for patients without cholecystectomy and $0.6 \%$ for patients with cholecystectomy).

Table 2. Recommended treatments by required treatment group for acute cholecystitis in the clinical practice guidelines.

\begin{tabular}{|c|c|c|c|c|c|c|}
\hline & \multicolumn{2}{|c|}{ GB drainage (-) } & \multicolumn{2}{|c|}{$\begin{array}{c}\text { GB drainage }(+) \\
\text { Supportive care }(-)\end{array}$} & \multicolumn{2}{|c|}{$\begin{array}{c}\text { GB drainage }(+) \\
\text { Supportive care }(+)\end{array}$} \\
\hline & $\begin{array}{l}\text { Cholecys- } \\
\text { tectomy (-) }\end{array}$ & $\begin{array}{l}\text { Cholecys- } \\
\text { tectomy }(+)\end{array}$ & $\begin{array}{l}\text { Cholecys- } \\
\text { tectomy (-) }\end{array}$ & $\begin{array}{l}\text { Cholecys- } \\
\text { tectomy }(+)\end{array}$ & $\begin{array}{l}\text { Cholecys- } \\
\text { tectomy (-) }\end{array}$ & $\begin{array}{l}\text { Cholecys- } \\
\text { tectomy }(+)\end{array}$ \\
\hline \multicolumn{7}{|l|}{ Recommendation A } \\
\hline (1) Administration of antimicrobial drugs (\%) & $\circ$ & ० & ० & $\circ$ & $\circ$ & ○ \\
\hline (2) Administration of NSAIDs (\%) & $\circ$ & ० & ○ & $\circ$ & $\circ$ & ० \\
\hline (3) Early cholecystectomy (within 4 days) (\%) & & ० & & $\circ$ & & ○ \\
\hline (4) Laparoscopic cholecystectomy (\%) & & o & & o & & o \\
\hline
\end{tabular}

GB, gallbladder; NSAIDs, nonsteroidal anti-inflammatory drugs; PTGBD, percutaneous transhepatic gallbladder drainage. 
The frequency of ENGBD was higher in patients with gallbladder drainage and without supportive care than those with both gallbladder drainage and supportive care, but not significantly so $(10.6 \%$ vs. $7.5 \%, p=0.241)$. The rate of bile culture performed in gallbladder drainage was significantly higher in patients with both gallbladder drainage and supportive care than those with gallbladder drainage and without supportive care $(88.3 \%$ vs. $76.3 \%, p=0.001)$. The proportion of patients with cholecystectomy was significantly higher in patients with both gallbladder drainage and supportive care than other patient groups. There was no significant difference with regard to the mean age, ratio of elderly patients, and gender proportion between groups. The highest proportion of patients with severe comorbid conditions was observed in patients with both gallbladder drainage and supportive care $(39.0 \%)$. The frequency of use of ICU was also significantly higher in patients with both gallbladder drainage and supportive care $(26.0 \%)$. This result suggests the appropriate use of the ICU according to the severity of acute cholecystitis. Significant variation of mean LOS was observed between patient groups. The in-hospital mortality of patients was significantly higher in patients with both gallbladder drainage and supportive care than other patient groups $(1.3 \%$ vs. $3.7 \%$ vs. $6.8 \%, p<0.001$, Table 3).

Rates of CPG-recommended treatments actually given are shown in Table 4. Antimicrobial drugs were used in a high proportion of all patient groups $(91.1 \%$ [without cholecystectomy] and $97.0 \%$ [with cholecystectomy] in patients without gallbladder drainage vs. $93.4 \%$ and $98.1 \%$ in those with gallbladder drainage and without supportive care vs. $98.7 \%$ and $98.5 \%$ in those with both gallbladder

Table 3. Clinical characteristics and presentations of patients according to required treatment group for acute cholecystitis.

\begin{tabular}{|c|c|c|c|c|}
\hline & GB drainage $(-)$ & $\begin{array}{c}\text { GB drainage }(+) \\
\text { Supportive care }(-)\end{array}$ & $\begin{array}{c}\text { GB drainage }(+) \\
\text { Supportive care }(-)\end{array}$ & $p$ value \\
\hline Number of patients & 4,333 & 1,591 & 146 & \\
\hline \multicolumn{5}{|l|}{ Gallbladder drainage (\%) } \\
\hline PTGBD & & 89.4 & 92.5 & 0.241 \\
\hline ENGBD & & 10.6 & 7.5 & \\
\hline Bile culture $(\%)$ & & 76.3 & 88.3 & 0.001 \\
\hline Patients with cholecystectomy (\%) & 36.1 & 30.9 & 45.8 & $<0.001$ \\
\hline Mean age (years (s.D.)) & $70.5(15.1)$ & $69.8(15.2)$ & $69.4(15.4)$ & 0.910 \\
\hline Elderly patients ( $\geq 75$ years) $(\%)$ & 43.9 & 41.6 & 44.5 & 0.270 \\
\hline Male patients $(\%)$ & 57.4 & 58.7 & 58.2 & 0.634 \\
\hline \multicolumn{5}{|l|}{ Chronic comorbid conditions (\%) } \\
\hline Mild & 55.6 & 49.5 & 38.4 & $<0.001$ \\
\hline Moderate & 21.5 & 22.1 & 22.6 & \\
\hline Severe & 22.9 & 28.4 & 39.0 & \\
\hline \multicolumn{5}{|l|}{ Hospital type (\%) } \\
\hline Academic hospitals & 8.6 & 9.9 & 13.0 & 0.072 \\
\hline Community hospitals & 91.4 & 90.1 & 87.0 & \\
\hline Use of ambulance transportation (\%) & 17.8 & 18.2 & 13.0 & 0.287 \\
\hline Use of intensive care unit (\%) & 3.9 & 3.8 & 26.0 & $<0.001$ \\
\hline Mean length of stay (days (s.D.)) & $17.0(14.1)$ & $26.5(22.0)$ & $45.0(35.5)$ & $<0.001$ \\
\hline In-hospital mortality (\%) & 1.3 & 3.7 & 6.8 & $<0.001$ \\
\hline
\end{tabular}

GB, gallbladder; s.D., standard deviation.

Table 4. Results of performance for recommended treatments for acute cholecystitis.

\begin{tabular}{|c|c|c|c|c|c|c|}
\hline & \multicolumn{2}{|c|}{ Without GB drainage } & \multicolumn{2}{|c|}{$\begin{array}{c}\text { GB drainage } \\
\text { without supportive care }\end{array}$} & \multicolumn{2}{|c|}{$\begin{array}{l}\text { GB drainage } \\
\text { with supportive care }\end{array}$} \\
\hline & $\begin{array}{l}\text { Cholecys- } \\
\text { tectomy (-) }\end{array}$ & $\begin{array}{l}\text { Cholecys- } \\
\text { tectomy }(+)\end{array}$ & $\begin{array}{l}\text { Cholecys- } \\
\text { tectomy (-) }\end{array}$ & $\begin{array}{l}\text { Cholecys- } \\
\text { tectomy }(+)\end{array}$ & $\begin{array}{l}\text { Cholecys- } \\
\text { tectomy (-) }\end{array}$ & $\begin{array}{l}\text { Cholecys- } \\
\text { tectomy }(+)\end{array}$ \\
\hline (1) Administration of antimicrobial drugs (\%) & 91.1 & 97.0 & 93.4 & 98.1 & 98.7 & 98.5 \\
\hline (2) Administration of NSAIDs (\%) & 39.6 & 66.9 & 56.2 & 88.0 & 60.7 & 76.1 \\
\hline (3) Early cholecystectomy (within 4 days) (\%) & & 50.7 & & 29.0 & & 46.2 \\
\hline (4) Laparoscopic cholecystectomy (\%) & & 63.7 & & 51.1 & & 31.3 \\
\hline
\end{tabular}

GB, gallbladder; NSAIDs, nonsteroidal anti-inflammatory drugs; PTGBD, percutaneous transhepatic gallbladder drainage. 
drainage and supportive care, respectively). Patients without gallbladder drainage received administration of NSAIDs less than other patient groups. Patients with gallbladder drainage and without supportive care least frequently underwent early cholecystectomy. Regarding early cholecystectomy, $59.5 \%$ of patients without gallbladder drainage underwent laparoscopic cholecystectomy whereas $71.0 \%$ of those with both gallbladder drainage and supportive care received open procedure (data not shown). Additionally, patients without gallbladder drainage were more likely to undergo laparoscopic cholecystectomy than other patient groups ( $63.7 \%$ vs. $51.1 \%$ vs. $31.3 \%$, respectively).

After adjusting for potential confounding effects of the clinical variables, multiple logistic regression analysis showed that patient group significantly influenced the performance of each recommended treatment. With regard to the administration of antimicrobial drugs, the odds ratio (OR) for patients with gallbladder drainage and without supportive care was 1.34 (95\% confidence interval (CI); $1.04-1.73, p=0.021)$, whereas the OR for those with both gallbladder drainage and supportive care was 4.38 (95\% CI; $1.07-17.87, p=0.039$ ). Regarding the administration of NSAIDs, the OR for patients with gallbladder drainage and without supportive care was 2.03 (95\% CI; $1.80-2.29, p<$ $0.001)$, whereas the OR for those with both gallbladder drainage and supportive care was 1.92 (95\% CI; 1.34-2.76, $p<0.001)$. Use of ICU was also associated with an increase in the administration of antimicrobial drugs and NSAIDs (OR 4.20 (95\% CI; 1.55-11.37, $p=0.005)$ and OR 1.88 (95\% CI; 1.44-2.47, $p<0.001)$, respectively). Otherwise, regarding early cholecystectomy, multiple logistic regression analysis also showed that patient group was significantly associated with decreased performance of early cholecystectomy. The OR for patients with gallbladder drainage and without supportive care was 0.39 (95\% $\mathrm{CI}$; 0.31-0.48, $p<0.001)$. However, no significant difference was seen for patients with both gallbladder drainage and supportive care (the OR for patients with both gallbladder drainage and supportive care was 0.75 (95\% CI; 0.45 $1.26, p=0.288)$. Regarding laparoscopic cholecystectomy, the OR for patients with gallbladder drainage and without supportive care was 0.59 (95\% CI; 0.48-0.73, $p<0.001$ ), whereas the OR for those with both gallbladder drainage and supportive care was 0.38 (95\% CI; 0.22-0.67, $p=0.001$; Table 5).

Table 5. Logistic regression analysis of each recommended treatments for acute cholecystitis.

\begin{tabular}{|c|c|c|c|c|c|c|c|c|c|c|c|c|}
\hline & \multicolumn{3}{|c|}{$\begin{array}{l}\text { Administration of } \\
\text { antimicrobial drugs }\end{array}$} & \multicolumn{3}{|c|}{$\begin{array}{l}\text { Administration of } \\
\text { NSAIDs }\end{array}$} & \multicolumn{3}{|c|}{ Early cholecystectomy } & \multicolumn{3}{|c|}{$\begin{array}{l}\text { Laparoscopic } \\
\text { cholecystectomy }\end{array}$} \\
\hline & OR & $95 \% \mathrm{CI}$ & $p$ value & OR & $95 \% \mathrm{CI}$ & $p$ value & OR & $95 \% \mathrm{CI}$ & $p$ value & OR & $95 \% \mathrm{CI}$ & $p$ value \\
\hline \multicolumn{13}{|l|}{ Procedures for acute cholecystitis } \\
\hline $\begin{array}{l}\text { GB drainage without supportive } \\
\text { care }\end{array}$ & 1.34 & $(1.04,1.73)$ & 0.021 & 2.03 & $(1.80,2.29)$ & $<0.001$ & 0.39 & $(0.31,0.48)$ & $<0.001$ & 0.59 & $(0.48,0.73)$ & $<0.001$ \\
\hline $\begin{array}{l}\text { GB drainage with supportive } \\
\text { care } \\
\text { (Reference; without GB drainage) }\end{array}$ & 4.38 & $(1.07,17.87)$ & 0.039 & 1.92 & $(1.34,2.76)$ & $<0.001$ & 0.75 & $(0.45,1.26)$ & 0.288 & 0.38 & $(0.22,0.67)$ & 0.001 \\
\hline \multicolumn{13}{|l|}{ Age } \\
\hline $\begin{array}{l}\text { Elderly patients } \\
\text { (Reference: younger patients) }\end{array}$ & 0.90 & $(0.72,1.12)$ & 0.357 & 1.52 & $(1.37,1.69)$ & $<0.001$ & 1.04 & $(0.87,1.25)$ & 0.634 & 0.89 & $(0.74,1.07)$ & 0.230 \\
\hline \multicolumn{13}{|l|}{ Sex } \\
\hline $\begin{array}{l}\text { Male } \\
\text { (Reference: female) }\end{array}$ & 0.95 & $(0.76,1.18)$ & 0.668 & 1.03 & $(0.92,1.14)$ & 0.578 & 1.01 & $(0.84,1.20)$ & 0.909 & 0.94 & $(0.78,1.13)$ & 0.562 \\
\hline \multicolumn{13}{|l|}{ Chronic comorbid conditions } \\
\hline Moderate & 0.92 & $(0.70,1.20)$ & 0.544 & 0.97 & $(0.85,1.10)$ & 0.686 & 0.69 & $(0.55,0.87)$ & 0.002 & 0.60 & $(0.48,0.76)$ & $<0.001$ \\
\hline $\begin{array}{l}\text { Severe } \\
\text { (Reference: mild) }\end{array}$ & 0.88 & $(0.68,1.14)$ & 0.365 & 0.90 & $(0.79,1.02)$ & 0.125 & 0.54 & $(0.43,0.69)$ & $<0.001$ & 0.57 & $(0.46,0.72)$ & $<0.001$ \\
\hline \multicolumn{13}{|l|}{ Hospital type } \\
\hline $\begin{array}{l}\text { Academic hospitals } \\
\text { (Reference: community hospitals) }\end{array}$ & 0.90 & $(0.63,1.29)$ & 0.581 & 1.07 & $(0.89,1.28)$ & 0.450 & 1.39 & $(1.04,1.85)$ & 0.023 & 1.07 & $(0.80,1.44)$ & 0.609 \\
\hline \multicolumn{13}{|l|}{ Ambulance transportation } \\
\hline $\begin{array}{l}\text { Use } \\
\text { (Reference: no use) }\end{array}$ & 1.09 & $(0.82,1.45)$ & 0.524 & 0.98 & $(0.86,1.13)$ & 0.851 & 0.89 & $(0.70,1.12)$ & 0.345 & 1.09 & $(0.86,1.39)$ & 0.445 \\
\hline \multicolumn{13}{|l|}{ Intensive care unit } \\
\hline $\begin{array}{l}\text { Use } \\
\text { (Reference: no use) }\end{array}$ & 4.20 & $(1.55,11.37)$ & 0.005 & 1.88 & $(1.44,2.47)$ & $<0.001$ & 1.78 & $(1.32,2.39)$ & $<0.001$ & 0.33 & $(0.24,0.45)$ & $<0.001$ \\
\hline
\end{tabular}




\section{Discussion}

We conducted this study to examine the management of acute cholecystitis for adherence to the appropriate CPGs using the administrative database associated with the DPC system. This study demonstrated that there were various differences with regard to the proportion of recommended treatments actually performed by the level of procedures required for treatment of acute cholecystitis, such as gallbladder drainage or supportive care.

In this study, bile culture was significantly more frequent in patients with both gallbladder drainage and supportive care, and antimicrobial drugs were more frequently received in accordance with the level of procedures required for treatment of acute cholecystitis. There is no doubt that the confirmation of systemic infection from acute cholecystitis and the identification of the causative agents were essential to patients who required gallbladder drainage or supportive care because systemic antibiotics are imperative when treating these patients, especially as some previous studies showed that a positive bile culture is correlated with the progression of acute cholecystitis (Pitt et al. 1983; Maluenda et al. 1989). In addition, more intensive care such as systemic antibiotics and supportive care is more necessary when the patient's condition is more severe, because these cases may be more likely to result in serious conditions such as organ dysfunction or septic shock. Therefore, clinicians are more likely to follow the recommendations for antimicrobial drugs in patients who required gallbladder drainage or supportive care. Also, the frequency of administration of NSAIDs was significantly higher in patients with gallbladder drainage or supportive care than in those without these procedures. A previous report suggested that NSAIDs relieved pain and prevented the progression of acute cholecystitis (Akriviadis et al. 1997). Therefore, it is also plausible that patients who required gallbladder drainage or supportive care had received NSAIDs more frequently.

On the contrary, regarding surgical treatments for acute cholecystitis, the rate of early cholecystectomy was lower in patients with gallbladder drainage and without supportive care, compared to other cases. Some reports have revealed that early cholecystectomy is safe and effective in patients with acute cholecystitis (Lo et al. 1998; Gurusamy et al. 2010). However, gallbladder drainage is also quite effective for controlling the inflammation of acute cholecystitis. Actually, CPGs for acute cholecystitis have demonstrated that elective cholecystectomy can be performed after the improvement of the acute inflammatory process by gallbladder drainage in moderate cholecystitis while mild or severe cholecystitis should require early cholecystectomy in the flowchart for the management of acute cholecystitis (Miura et al. 2007). Therefore, many clinicians may not consider early cholecystectomy due to their expectation of the effect of gallbladder drainage in moderate cholecystitis while they tend to decide to perform early cholecystectomy without hesitation in severe acute cholecystitis because it is a systemic disease. In addition, the frequency of laparoscopic cholecystectomy decreased as the level of required procedures increased for acute cholecystitis. Indeed, laparoscopic cholecystectomy is preferable to open procedures in patients with acute cholecystitis (Zacks et al. 2002; Peng et al. 2005; Catani and Modini 2007). However, the efficacy of this surgical management remains controversial in patients with pan-peritonitis or those with severe concomitant disease (Yamashita et al. 2007). In addition, Borzellino et al. (2008) reported that a lower feasibility of laparoscopic cholecystectomy was found for severe cholecystitis in their meta-analysis. In this study, we confirmed that most of cholecystectomies for patients with both gallbladder drainage and supportive care were performed by open procedures, not laparoscopic procedures. Therefore, this result suggests that many surgeons may give less priority to laparoscopic cholecystectomy for patients that required gallbladder drainage or supportive care. To resolve these discrepancies, further clinical trials examining the association of laparoscopic cholecystectomy with patient condition during hospitalization are needed.

The clinical data used represent a major strength of the current study. Acute cholecystitis is usually treated in acute care hospitals. One of the benefits of the national database was that it enabled evaluation of a large number of acute care hospitals in an unbiased manner, because our investigation involved a nationally representative sample of patients with acute cholecystitis in a community setting (Murata et al. 2010, 2011, 2012). Therefore, the data from this administrative database closely reflect the clinical circumstances of the procedures and treatments for acute cholecystitis. In addition, detailed medical data such as all procedures, medications and devices have been exhaustively coded with Japanese original payment codes (Murata et al. 2010, 2011, 2012). These data were recorded on a daily basis for each patient (Murata et al. 2010, 2011, 2012). Therefore, this administrative database also enables interested parties to evaluate the care processes according to the CPGs with individual detailed medical treatments.

A limitation of this study also warrants mention. The CPGs determine the severity of acute cholecystitis based on the laboratory data and imaging findings of patients (Mayumi et al. 2007). Regrettably, laboratory and imaging data are not recorded in the Japanese administrative database (Murata et al. 2010, 2011, 2012). Our categorization of patients by way of procedures performed for acute cholecystitis may thus have led to bias in the classification of cases. Therefore, further clinical studies evaluating the care processes according to the CPGs for acute cholecystitis are required, using stricter criteria to define the severity of acute cholecystitis.

Despite this limitation, the current study confirmed that the rate of appropriate treatments recommended by the CPGs increased in accordance with the level of procedures required for treatments of acute cholecystitis. Our study 
has significant implications for health care policy decisionmaking. First, the evaluation of the care processes according to the CPGs could be useful data for revealing the discrepancies between actual medical treatment and CPGs. Some previous reports suggest that the monitoring of the care processes according to the CPGs is useful evidence for maintaining and improving the quality of medical care as a measure of quality improvement (Quaglini et al. 2004; Gulati et al. 2004). Thus, we think that such study can reveal the difference between actual medical treatments and CPGs and favorably influence the further improvement and reconsideration of CPGs. Second, until recently, available data had been limited for such clinical epidemiological studies in Japan because there had been no large administrative databases, unlike in many European countries or the United States (Yasunaga and Horiguchi 2010). However, the administrative database associated with the DPC system contains detailed medical data on numerous patients. Therefore, we believe that this administrative database will allow the conduct of various large-scale clinical epidemiological studies in Japan, such as the evaluation of the care processes recommended by CPGs for various other diseases as well as acute cholecystitis, since the principal diagnosis and comorbidities of patients are strictly determined by the ICD-10th code and recorded (Murata et al. 2011; Ichimiya et al. 2011). Thus, the administrative database associated with the DPC system is a feasible tool that will provide useful information contributing to improve quality of medical care for various diseases in the near future.

In conclusion, we have demonstrated that there were various differences with regard to the proportion of appropriate CPG-recommended treatments actually performed by the level of procedures required for treatment of acute cholecystitis. In addition, the administrative database associated with the DPC system was a feasible tool for the evaluation of adherence to recommended CPGs care processes. Further the evaluation of the care processes according to CPGs using this administrative database will be actively conducted for various diseases and these studies will provide useful information contributing to improved quality of medical care in the near future.

\section{Acknowledgments}

This study was funded by Grants-in-Aid for Research on Policy Planning and Evaluation from the Ministry of Health, Labour and Welfare, Japan.

\section{Conflict of Interest}

All authors have no conflict of interest to declare.

\section{References}

Akhan, O., Akinci, D. \& Ozmen, M.N. (2002) Percutaneous cholecystostomy. Eur. J. Radiol., 43, 229-236.

Akriviadis, E.A., Hatzigavriel, M., Kapnias, D., Kirimlidis, J., Markantas, A. \& Garyfallos, A. (1997) Treatment of biliary colic with diclofenac: a randomized, double-blind, placebocontrolled study. Gastroenterology, 113, 225-231.
Barie, P.S. \& Eachempati, S.R. (2010) Acute acalculous cholecystitis. Gastroenterol. Clin. North Am., 39, 343-357.

Borzellino, G., Sauerland, S., Minicozzi, A.M., Verlato, G., Di Pietrantonj, C., de Manzoni, G. \& Cordiano, C. (2008) Laparoscopic cholecystectomy for severe acute cholecystitis. A meta-analysis of results. Surg. Endosc., 22, 8-15.

Catani, M. \& Modini, C. (2007) Laparoscopic cholecystectomy in acute cholecystitis: a proposal of safe and effective technique. Hepatogastroenterology, 54, 2186-2191.

Gulati, M., Patel, S., Jaffe, A.S., Joseph, A.J. \& Calvin, J.E. Jr. (2004) Impact of contemporary guideline compliance on risk stratification models for acute coronary syndromes in The Registry of Acute Coronary Syndromes. Am. J. Cardiol., 94, 873-878

Gurusamy, K., Samraj, K., Gluud, C., Wilson, E. \& Davidson, B.R. (2010) Meta-analysis of randomized controlled trials on the safety and effectiveness of early versus delayed laparoscopic cholecystectomy for acute cholecystitis. Br. J. Surg., 97, 141-150.

Ichimiya, Y., Matsuda, S., Fujino, Y., Kubo, T., Murata, A., Kuwabara, K., Fujimori, K. \& Horiguchi, H. (2011) Profiling of the care processes for laryngeal cancer with the Japanese administrative database. Tohoku. J. Exp. Med., 223, 61-66.

Kimura, Y., Takada, T., Kawarada, Y., Nimura, Y., Hirata, K., Sekimoto, M., Yoshida, M., Mayumi, T., Wada, K., Miura, F., Yasuda, H., Yamashita, Y., Nagino, M., Hirota, M., Tanaka, A., Tsuyuguchi, T., Strasberg, S.M. \& Gadacz, T.R. (2007) Definitions, pathophysiology, and epidemiology of acute cholangitis and cholecystitis: Tokyo Guidelines. J. Hepatobiliary Pancreat. Surg., 14, 15-26.

Kitano, S., Matsumoto, T., Aramaki, M. \& Kawano, K. (2002) Laparoscopic cholecystectomy for acute cholecystitis. $J$. Hepatobiliary Pancreat. Surg., 9, 534-537.

Kubo, T., Fujino, Y., Murata, A., Ichimiya, Y., Kuwabara, K., Fujimori, K., Horiguchi, H. \& Matsuda, S. (2011) Prevalence of type 2 diabetes among acute inpatients and its impact on length of hospital stay in Japan. Intern. Med., 50, 405-411.

Lee, S.W., Chang, C.S., Lee, T.Y., Tung, C.F. \& Peng, Y.C. (2010) The role of the Tokyo guidelines in the diagnosis of acute calculous cholecystitis. J. Hepatobiliary Pancreat. Surg., 17, 879-884.

Lo, C.M., Liu, Cl., Fan, S.T., Lai, E.C. \& Wong, J. (1998) Prospective randomized study of early versus delayed laparoscopic cholecystectomy for acute cholecystitis. Am. Surg., 227, 461-467.

Maluenda, F., Csendes, A., Burdiles, P. \& Diaz, J. (1989) Bacteriological study of choledochal bile in patients with common bile duct stones, with or without acute suppurative cholangitis. Hepatogastroenterology, 36, 132-135.

Matsuda, S. (2008) Casemix as a tool for transparency of medical services. Jpn. J. Soc. Security Policy, 6, 43-53.

Mayumi, T., Takada, T., Kawarada, Y., Nimura, Y., Yoshida, M., Sekimoto, M., Miura, F., Wada, K., Hirota, M., Yamashita, Y., Nagino, M., Tsuyuguchi, T., Tanaka, A., Gomi, H. \& Pitt, H.A. (2007) Results of the Tokyo Consensus Meeting Tokyo Guidelines. J. Hepatobiliary Pancreat. Surg., 14, 114-121.

Miura, F., Takada, T., Kawarada, Y., Nimura, Y., Wada, K., Hirota, M., Nagino, M., Tsuyuguchi, T., Mayumi, T., Yoshida, M., Strasberg. S.M., Pitt, H.A., Belghiti, J., de Santibanes, E., Gadacz. T.R., Gouma. D.J., Fan, S.T., Chen, M.F., Padbury, R.T., Bornman, P.C., Kim, S.W., Liau, K.H., Belli, G. \& Dervenis, C. (2007) Flowcharts for the diagnosis and treatment of acute cholangitis and cholecystitis: Tokyo Guidelines. J. Hepatobiliary Pancreat. Surg., 14, 27-34.

Murata, A., Matsuda, S., Kuwabara, K., Fujino, Y., Kubo, T., Fujimori, K. \& Horiguchi, H. (2010) Impact of hospital volume on clinical outcomes of endoscopic biliary drainage for acute cholangitis based on the Japanese administrative database associated with the Diagnosis Procedure Combina- 
tion system. J. Gastroenterol., 45, 1090-1096.

Murata, A., Matsuda, S., Mayumi, T., Yokoe, M., Kuwabara, K., Ichimiya, Y., Fujino, Y., Kubo, T., Fujimori, K. \& Horiguchi, H. (2011) A descriptive study evaluating the circumstances of medical treatment for acute pancreatitis before publication of the new JPN guidelines based on the Japanese administrative database associated with the Diagnosis Procedure Combination system. J. Hepatobiliary Pancreat. Surg., 18, 678-683.

Murata, A., Matsuda, S., Mayumi, T., Okamoto, K., Kuwabara, K., Ichimiya, Y., Fujino, Y., Kubo, T., Fujimori, K. \& Horiguchi, H. (2012) Multivariate analysis of factors influencing medical costs of acute pancreatitis hospitalizations based on a national administrative database. Dig. Liver Dis., 44, 143-148.

Navez, B., Mutter, D., Russier, Y., Vix, M., Jamali, F., Lipski, D., Cambier, E., Guiot, P., Leroy, J. \& Marescaux, J. (2001) Safety of laparoscopic approach for acute cholecystitis: retrospective study of 609 cases. World J. Surg., 25, 1352-1356.

Peng, W.K., Sheikh, Z., Nixon, S.J. \& Paterson-Brown, S. (2005) Role of laparoscopic cholecystectomy in the early management of acute gallbladder disease. Br. J. Surg., 92, 586-591.

Pitt, H.A., Postier, R.G. \& Cameron, J.L. (1983) Consequences of preoperative cholangitis and its treatment on the outcome of operation for choledocholithiasis. Surgery, 94, 447-452.

Quaglini, S., Cavallini, A., Gerzeli, S. \& Micieli, G. (2004) Economic benefit from clinical practice guideline compliance in stroke patient management. Health Policy, 69, 305-315.

Quan, H., Sundararajan, V., Halfon, P., Fong, A., Burnand, B., Luthi, J.C., Saunders, L.D., Beck, C.A., Feasby, T.E. \& Ghali, W.A. (2005) Coding algorithms for defining comorbidities in ICD-9-CM and ICD-10 administrative data. Med. Care, 43, $1130-1139$.

Takada, T., Kawarada, Y., Nimura, Y., Yoshida, M., Mayumi, T., Sekimoto, M., Miura, F., Wada, K., Hirota, M., Yamashita, Y., Nagino, M., Tsuyuguchi, T., Tanaka, A., Kimura, Y., Yasuda, H., Hirata, K., Pitt, H.A., Strasberg, S.M., Gadacz, T.R., Bornman, P.C., Gouma, D.J., Belli, G. \& Liau, K.H. (2007) Background: Tokyo Guidelines for the management of acute cholangitis and cholecystitis. J. Hepatobiliary Pancreat. Surg., 14, 1-10.

Toyota, N., Takada, T., Amano, H., Yoshida, M., Miura, F. \& Wada,
K. (2006) Endoscopic naso-gallbladder drainage in the treatment of acute cholecystitis: alleviates inflammation and fixes operator's aim during early laparoscopic cholecystectomy. $J$. Hepatobiliary Pancreat. Surg., 13, 80-85.

Yamashita, Y., Takada, T., Kawarada, Y., Nimura, Y., Hirota, M., Miura, F., Mayumi, T., Yoshida, M., Strasberg, S., Pitt, H.A., de Santibanes, E., Belghiti, J., Büchler, M.W., Gouma, D.J., Fan, S.T., Hilvano, S.C., Lau, J.W., Kim, S.W., Belli, G., Windsor, J.A., Liau, K.H. \& Sachakul, V. (2007) Surgical treatment of patients with acute cholecystitis: Tokyo Guidelines. J. Hepatobiliary Pancreat. Surg., 14, 91-97.

Yasuda, H., Takada, T., Kawarada, Y., Nimura, Y., Hirata, K., Kimura, Y., Wada, K., Miura, F., Hirota, M., Mayumi, T., Yoshida, M., Nagino, M., Yamashita, Y., Hilvano, S.C. \& Kim, S.W. (2007) Unusual cases of acute cholecystitis and cholangitis: Tokyo Guidelines. J. Hepatobiliary Pancreat. Surg., 14, 98-113.

Yasunaga, H. \& Horiguchi, H. (2010) Clinical epidemiological studies using the Diagnosis Procedure Combination Database. Iryo to Shakai, 20, 87-96 (in Japanese).

Yokoe, M., Takada, T., Mayumi, T., Yoshida, M., Hasegawa, H., Norimizu, S., Hayashi, K., Umemura, S. \& Orito, E. (2011) Accuracy of the Tokyo Guidelines for the diagnosis of acute cholangitis and cholecystitis taking into consideration the clinical practice pattern in Japan. J. Hepatobiliary Pancreat. Surg., 18, 250-257.

Yoshida, M., Takada, T., Kawarada, Y., Tanaka, A., Nimura, Y., Gomi, H., Hirota, M., Miura, F., Wada, K., Mayumi, T., Solomkin, J.S., Strasberg, S., Pitt, H.A., Belghiti, J., de Santibanes, E., Fan, S.T., Chen, M.F., Belli, G., Hilvano, S.C., Kim, S.W. \& Ker, C.G. (2007) Antimicrobial therapy for acute cholecystitis: Tokyo Guidelines. J. Hepatobiliary Pancreat. Surg., 14, 83-90.

Westphal, J.F. \& Brogard, J.M. (1999) Biliary tract infections: a guide to drug treatment. Drugs, 57, 81-91.

Zacks, S.L., Sandler, R.S., Rutledge, R. \& Brown, R.S. Jr. (2002) A population based cohort study comparing laparoscopic cholecystectomy and open cholecystectomy. Am. J. Gastroenterol., 97, 334-340. 